\title{
Applied Nanopore Fabrication Techniques for DNA and Protein Sensing
}

\author{
Cuifeng Ying \\ Advanced Optics \& Photonics Laboratory, Department of Engineering, School of Science \& Technology, \\ Nottingham Trent University, Nottingham NG11 8NS, UK \\ cuifeng.ying@ntu.ac.uk
}

\section{Extended Abstract}

In the past two decades, solid-state nanopores have become a popular tool for the detection and characterisation of biomolecules such as DNA, proteins, and virus. The basic experimental setup for nanopore sensing comprises two compartments of electrolyte solution connected solely by a single pore with a diameter ranging from 1-50 nm. Nanopore techniques measure the flux of ions in a nanopore within a detection volume of $10^{-24}$ litres and can detect biomolecules in the nanomolar concentration range. A target molecule diffusing into the nanopore excludes some of the conducting ions, resulting in a measurable ionic current blockade that directly corresponds to the molecule's volume and residence time. Compared to biological nanopores, solid-state nanopores are garnering increasing interest due to their robustness and their potential to be fabricated at virtually any size below $1 \mu \mathrm{m}$.

While current nanopore fabrication processes using ion or electron beam drilling can produce nanopores with 1-nm precision, these methods often require advanced skill and specialized instrumentation, limiting broad access. In this presentation, I summarize several of our research works on nanopore fabrication in the attempts to address challenges in DNA and protein sensing [1-6]. It will be shown that controlled breakdown can generate cone-shaped nanopores with precise size control of 2 to $4 \mathrm{~nm}$.[2] These nanopores are able to discriminate 20-nucleotide single-stranded DNA composed of homopolymers (poly $(\mathrm{dA})_{20}$, poly $(\mathrm{dC})_{20}$, and poly $(\mathrm{dT})_{20}$ ) by their ionic current signals. Analysing and classifying proteins using nanopores, on the other hand, comes with different challenges due to their wide size distribution ranging from $1 \mathrm{~nm}$ to $100 \mathrm{~nm}$. The attempts to fabricate large nanopores with diameters exceeding $\sim 20 \mathrm{~nm}$ via breakdown often result in the undesirable formation of multiple nanopores in $\mathrm{SiN}_{\mathrm{x}}$ membranes.[3] To address this issue, we employed laser-induced breakdown (LBD) to improve the probability of formation of single nanopores with diameters of 20-50 nm.[4] In addition to the problem of pore size, protein detection was limited to low bandwidth measurements due to high signal noise, limiting the ability to determine the conformation of proteins that translocate quickly. To mitigate the detrimental effects of signal noise, we increased the recording bandwidth by designing and fabricating glass nanopores that exhibit low noise characteristics, resolving protein translocation signals using up to $1 \mathrm{MHz}$ bandwidth recordings without compromising signal-to-noise ratio.[5] By conducting a rigorous analysis process, we have demonstrated that data gathered from a nanopore can discriminate proteins based on their size, shape and dipole moment without any tethering.[6] We believe that these approaches of generating nanopores for various applications will further accelerate the development of nanopore-based characterization of single molecules.

\section{References}

[1] Y. Feng, Y. Zhang, C. Ying, D. Wang, and C. Du, "Nanopore-based fourth-generation DNA sequencing technology," Genomics, proteomics \& bioinformatics, vol. 13, no. 1, pp. 4-16, 2015.

[2] C. Ying, Y. Zhang, Y. Feng, D. Zhou, Y. Chen, C. Du, D. Wang, J. Tian, "3D nanopore shape control by current-stimulus dielectric breakdown," Appl Phys Lett, vol. 109, no. 6, p. 063105, 2016.

[3] Y. Wang, C. Ying, W. Zhou, L. de Vreede, Z. Liu, and J. Tian, "Fabrication of multiple nanopores in a SiNx membrane via controlled breakdown," Sci Rep, vol. 8, no. 1, p. 1234, 2018.

[4] C. Ying, J. Houghtaling, O. M. Eggenberger, A. Guha, P. Nirmalraj, S. Awasthi, J. Tian, M. Mayer, "Formation of Single Nanopores with Diameters of 20-50 nm in Silicon Nitride Membranes Using Laser-Assisted Controlled Breakdown," ACS Nano, vol. 12, no. 11, pp. 11458-11470, 2018 
[5] L. de Vreede, C. Ying, J. Houghtaling, J. Figueiredo Da Silva, A. Hall, A. Lovera, M. Mayer, "Wafer scale fabrication of fused silica chips for low-noise recording of resistive pulses through nanopores," Nanotechnology, vol. 30, no. 26, p. 265301, 2019.

[6] J. Houghtaling, C. Ying, O. Eggenberger, A. Fennouri, S. Nanivida, M. Acharjee, J. Li; A. Hall, M. Mayer, "Estimation of Shape, Volume, and Dipole Moment of Individual Proteins Freely Transiting a Synthetic Nanopore," ACS Nano, vol. 13 , no. 5, pp. 5231-5242, 2019 